\title{
Infrared Absorption Spectroscopy of Monolayers with Thin Film Interference Coatings
}

\author{
Sencer Ayas $\left[{ }^{* a, b}\right]$, Gokhan Bakan $\left[{ }^{\mathrm{a}, \mathrm{c}}\right]$, Erol Ozgur $\left[{ }^{\mathrm{a}}\right]$, Kemal Celebi $\left[{ }^{\mathrm{a}}\right]$ and and Aykutlu Dana $\left[^{\mathrm{a}}\right]$ \\ ${ }^{\mathrm{a}}$ UNAM, Institute of Materials Science and Nanotechnology, Bilkent University, Ankara, 06800, Turkey.

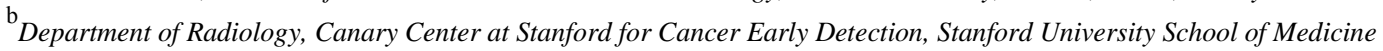 \\ ${ }^{\mathrm{c}}$ Department of Electrical and Electronics Engineering, Atilim University, Ankara, 06830, Turkey \\ *sencerayas@gmail.com
}

\begin{abstract}
We report high performance Infrared spectroscopy platforms based on interference coatings on metal using $\mathrm{CaF}_{2}$ dielectric films and $\mathrm{Ge}_{2} \mathrm{Sb}_{2} \mathrm{Te}_{5}$ (GST) phase-change films. IR vibrational bands of proteins and organic monolayers are also detected.

OCIS codes: (300.0300) Spectroscopy; (240.0240) Optics at surfaces
\end{abstract}

\section{Introduction}

Infrared absorption spectroscopy is a powerful method to directly probe the vibrational signatures of molecules, enabling label-free biochemical analysis[1], [2]. However, because of the poor interaction between the infrared field and nanoscale molecules, conventional infrared absorption methods require large numbers of molecules to collect significant information, making them insufficient to detect thin films or monolayers, which is crucial for biomolecular sensing. Plasmonic field enhancement by surface nanostructuring has been widely studied in recent years, enabling the technique called Surface Enhanced Infrared Absorption Spectroscopy (SEIRA) [3]-[6]. However, as other plasmonic enhancement methods, SEIRA also requires patterned plasmonic structures that provide optical resonances close to the vibrational modes of the molecules, narrowing down the detection bandwidth. Hence, the resonance wavelength of the plasmonic structure has to be tuned [5], [7]. To overcome the need for tuning by increasing the bandwidth, spatially multiplexed designs with multispectral responses have also been demonstrated[4]. Here, we propose thin film structures for IR sensing and demonstrate the advantages of such structures over onventional plasmonic structures (SEIRA), in terms of fabrication, signal intensity, bandwidth and spatial detection range. We also present IR-based detection of self-assembled monolayers and proteins on these platforms[8], [9].
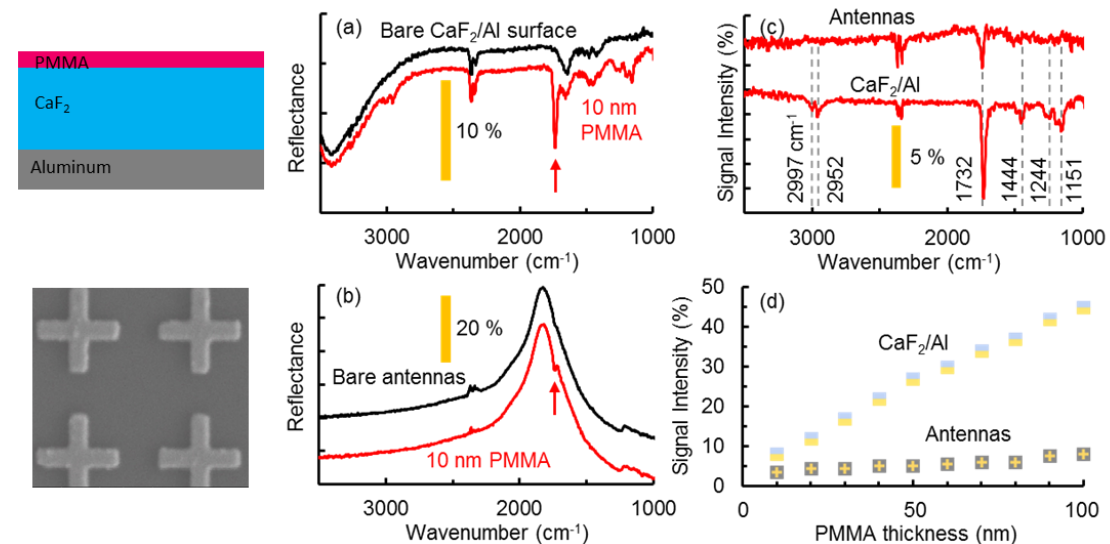

Fig 1. Experimental comparison of the $\mathrm{CaF}_{2} / \mathrm{Al}$ surface to the plasmonic antenna-array, for IR spectroscopy. Reflection spectra of bare and $10 \mathrm{~nm}$ PMMA coated $\mathrm{CaF}_{2} / \mathrm{Al}$ surface (a), and plasmonic antenna surface (b). (c) Background subtracted signal intensities. (d) The thickness dependent comparison of $\mathrm{CaF}_{2} / \mathrm{Al}$ and plasmonic antenna-array.

\section{Results}

Figure 1 shows the infrared absorption spectroscopy performances of the thin film surfaces and plasmonic structures. We have selected PMMA as the probe material, $\mathrm{Al}$ as the reflector, $\mathrm{CaF}_{2}$ as the dielectric and $\mathrm{Ag}$ to form the plasmonic antennas that are defined by e-beam lithography on silicon. The thickness of $\mathrm{CaF}_{2}$ is chosen as 900 $\mathrm{nm}$, to have a field enhancement above unity at the IR range of $\lambda: 3-12 \mu \mathrm{m}$. Thus, the reflection spectrum without PMMA is mainly flat above $3200 \mathrm{~cm}^{-1}$, as expected by the simulations, except the dips at 3400 and $1640 \mathrm{~cm}^{-1}$, which can be attributed to the O-H modes (Fig.1a). This broad bandwidth enables easy identification of all the PMMA bands ranging from 3000 to $1000 \mathrm{~cm}^{-1}$, even when there is a very thin layer of PMMA on $\mathrm{CaF}_{2}$ (Fig.1a). 
The reflection from the 10-nm-thick PMMA coated antennas, however, only shows the major PMMA peak at 1732 $\mathrm{cm}^{-1}$ (Fig.1b) with lower signal intensity (3.5\% vs. $7.7 \%$ ) as shown in the background-subtracted data in (Fig.1c). Besides the bandwidth and signal intensity, the spatial extent of the field enhancement by the $\mathrm{CaF}_{2}$ surfaces is much larger. Thus, when a thicker (100 nm) layer of PMMA is coated on both surfaces, the dip at $1732 \mathrm{~cm}^{-1}$ reaches $45 \%$ of the total reflection for the $\mathrm{CaF}_{2}$ surface, while the same dip is a mere $8 \%$ for the antenna surface (Fig.1d).

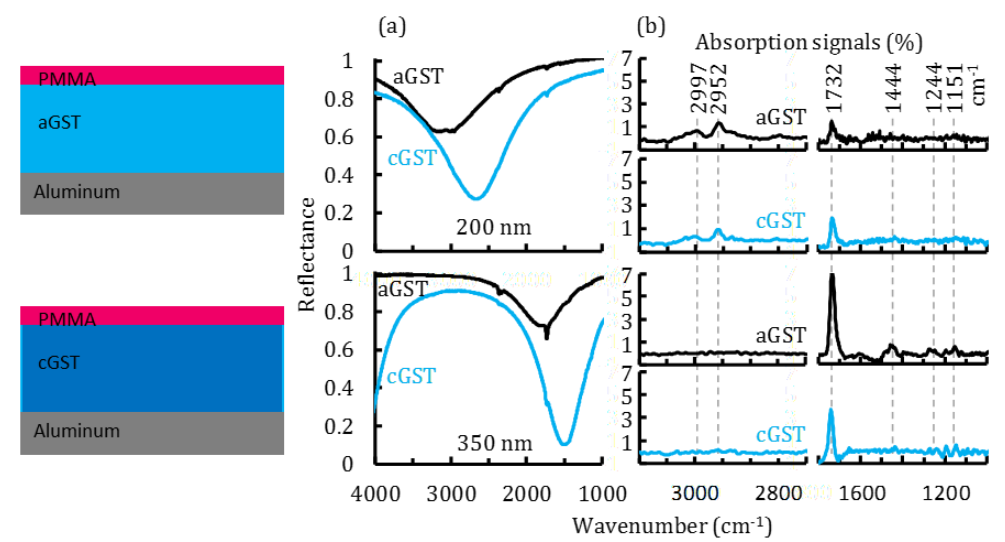

Fig 2. Infrared absorption spectroscopy of $10 \mathrm{~nm}$ PMMA films on GST/Al surfaces. (a) Reflection spectra of $10 \mathrm{~nm}$ PMMA coated aGST/Al and cGST/Al surfaces. 200 and $350 \mathrm{~nm}$ GST thicknesses are used to address PMMA's lower and higher wavelength absorption bands. (b) Absorption of the PMMA layers extracted from the reflection spectra in (a). The major PMMA absorption bands are highlighted with dashed lines. .

In Fig 2, we have demonstrated a thermally tunable IR spectroscopy platform based on GST phase-change films on $\mathrm{Al}$ mirror. The infrared absorption sensing performance of the fabricated surfaces are tested using $10 \mathrm{~nm}$ thick PMMA layers. The GST thicknesses are chosen as 200 and $350 \mathrm{~nm}$ targeting the PMMA vibrational bands around 3000 and $1500 \mathrm{~cm}^{-1}$, respectively. The PMMA absorption bands are observed as narrow dips on the reflection spectra (Fig. 2a). aGST (200 nm)/Al surface can sense the PMMA absorption bands at 2997, 2952 and $1732 \mathrm{~cm}^{-1}$. Crystallization of the GST layer, prior to coating the PMMA layer, lowers the absorption at $2997,2952 \mathrm{~cm}^{-1}$ and enhances the absorption at 1732 and $1444 \mathrm{~cm}^{-1}$ (Fig. 2b). The major PMMA band at $1732 \mathrm{~cm}^{-1}$ is observed with a signal intensity of $\sim 7 \%$ on aGST $(350 \mathrm{~nm}$ )/Al surface. This surface is particularly good at sensing all the vibrational bands between 1732 and $1151 \mathrm{~cm}^{-1}$. On cGST(350 nm)/Al surface, although the signal intensity for $1732 \mathrm{~cm}^{-1}$ band drops to $3.5 \%$, the higher wavelength absorption bands $\left(1192-754 \mathrm{~cm}^{-1}\right)$ appear as clear peaks.

\section{Conclusion}

In conclusion, we have demonstrated broadband $\left(\mathrm{CaF}_{2} / \mathrm{Al}\right)$ and tunable IR spectroscopy platform using interference coatings on metal mirrors. The proposed surfaces outperform plasmonic counterparts in terms of signal enhancement, bandwidth and spatial extend of field.

\section{References}

[1] A. Hartstein, J. R. Kirtley, and J. C. Tsang, "Enhancement of the Infrared Absorption from Molecular Monolayers with Thin Metal Overlayers," Phys. Rev. Lett., vol. 45, no. 3, pp. 201-204, Jul. 1980.

[2] C. Kendall et al., "Vibrational spectroscopy: a clinical tool for cancer diagnostics.," Analyst, vol. 134, no. 6, pp. $1029-45$, Jun. 2009.

[3] R. Adato et al., "Ultra-sensitive vibrational spectroscopy of protein monolayers with plasmonic nanoantenna arrays.," Proc. Natl. Acad. Sci. U. S. A., vol. 106, no. 46, pp. 19227-32, Nov. 2009.

[4] H. Aouani et al., "Plasmonic Nanoantennas for Multispectral Surface-Enhanced Spectroscopies," J. Phys. Chem. C, vol. 117, no. 36, pp. 18620-18626, Sep. 2013.

[5] L. V Brown, K. Zhao, N. King, H. Sobhani, P. Nordlander, and N. J. Halas, "Surface-enhanced infrared absorption using individual cross antennas tailored to chemical moieties.," J. Am. Chem. Soc., vol. 135, no. 9, pp. 3688-95, Mar. 2013.

[6] R. Adato and H. Altug, "In-situ ultra-sensitive infrared absorption spectroscopy of biomolecule interactions in real time with plasmonic nanoantennas.," Nat. Commun., vol. 4, p. 2154, Jan. 2013.

[7] D. Rodrigo et al., "Mid-infrared plasmonic biosensing with graphene," Science (80-. )., vol. 349, no. 6244, pp. 165-168, Jul. 2015.

[8] S. Ayas, G. Bakan, E. Ozgur, K. Celebi, and A. Dana, "Universal Infrared Absorption Spectroscopy Using Uniform Electromagnetic Enhancement," ACS Photonics, vol. 3, no. 3, pp. 337-342, Mar. 2016.

[9] G. Bakan, S. Ayas, E. Ozgur, K. Celebi, and A. Dana, "Thermally Tunable Ultrasensitive Infrared Absorption Spectroscopy Platforms Based on Thin Phase-Change Films," ACS Sensors, Nov. 2016. 